Rev. Elev. Méd. vét. Pays trop., 1973, 26 (4) : 49 a - 60 a

\title{
Caractéristiques de la reproduction chez le zébu étudiées au centre de recherches zootechniques de Dahra
}

\author{
par J. DENIS (*) et A. I. THIONGANE $\left(^{* *}\right)$
}

\begin{abstract}
RESUME
Dans ce rapport, il est tenté de faire le point sur les diverses caractéristiques actuellement connues de la reproduction chez le zébu Gobra. Sont analysés successivement l'âge aú 1er vêlage, l'intervalle entre les vêlages, les problèmes posés par l'installation d'une saison de monte, la durée de la gestation, l'âge de la puberté, les chaleurs (intervalles et durée), l'involution utérine, le problème de la maîtrise des cycles ostriens. L'étude de certains facteurs influençant la vie de reproduction tels que la saison, la nutrition et la lactation est ensuite abordée. Enfin, on donne quelques informations sur les mâles.
\end{abstract}

\section{INTRODUCTION}

Les phénomènes concernant la reproduction sont en général assez mal connus chez les zébus africains. Le zébu Gobra du Sénégal n'échappe pas à ce fait et pourtant, dans le domaine de l'élevage et de son amélioration, il est nécessaire d'avoir une juste connaissance de ces phénomènes.

L'étude de la sexualité a été systématiquement entreprise au Centre de Recherches zootechniques de Dahra-Djoloff aussi bien chez les mâles que chez les femelles.

Il est bien évident que les conditions d'existence du zébu Gobra au C.R.Z. sont un progrès par rapport à celles rencontrées dans la région d'extension de la race. Malheureusement, les données concernant la reproduction chez les animaux en brousse sont extrêmement peu nombreuses et, en fait, sont plutôt des estimations que des certitudes. C'est pourquoi les données recueillies en station conservent toute leur valeur, surtout qu'en principe les améliorations apportées aux conditions de vie ne sont pas telles qu'il existe une véritable coupure entre les modes d'entretien des animaux.

La connaissance du déroulement de la vie de reproduction, en particulier chez la femelle, est nécessaire pour essayer d'agir sur les différents facteurs en vue de l'amélioration du rendement animal et en particulier permet d'envisager l'utilisation de la technique de l'insémination artificielle. En effet, cette opération permet de multiplier le pouvoir de fécondation des mâles sélectionnés, testés et entretenus dans les meilleures conditions possibles au C.R.Z. de Dahra, alors que les opérations actuelles de vulgarisation doivent faire intervenir un nombre de tau-

(*) I.E.M.V.T., Centre de Recherches Zootechniques de Dahra, Sénégal. 
reaux beaucoup plus important, donc de valeur moyenne plus faible et entretenus dans des conditions parfois très précaires.

Dans ce rapport, l'ensemble des données actuellement disponibles sur le zébu Gobra sera présenté. Sur certains points, les connaissances sont solides et basées sur de nombreuses observations. Sur d'autres, les observations systématiques sont récentes et par conséquent les valeurs exprimées seront sujettes à révision ou plutôt à réajustement au fur et à mesure de l'accumulation des résultats.

\section{LE MILIEU}

L'influence du climat en général sur les comportements animaux n'est plus à démontrer. Par contre, ce qui fait la particularité des milieux difficiles et en particulier des milieux arides, c'est que le facteur climatique n'a pas une influence nuancée mais se comporte à certaines périodes comme un véritable facteur limitant. Dans la région écologique qui nous intéresse, les animaux ressentent durement dans l'expression de leurs diverses performances l'action météorologique.

C'est pourquoi, au seuil de cette étude, il convient de préciser ces divers facteurs, pour en apprécier les conséquences sur les animaux présentés plus avant.

Le climat du Sénégal est du type tropical sec. Il existe au cours de l'année deux saisons bien tranchées:

- une saison sèche durant de 7 à 9 mois. Les mois le plus secs sont mars et avril;

- une saison des pluies durant de 3 à 5 mois. Les mois les plus pluvieux sont août et septembre.

Le Centre de Recherches zootechniques est situé à la croisée des degrés $14^{\circ} 5$ de latitude Nord et de longitude Ouest. La station climatique la plus proche est celle de Linguère dont les caractéristiques sont représentatives de celles de la zone sylvo-pastorale dans laquelle évoluent les animaux Gobra.

Le Centre possède un petit poste qui permet de relever localement la température, la pluviométrie et depuis deux années l'hygrométrie.

Mais ce sont les caractéristiques générales de la région (station de Linguère) qui seront exposées.

\section{La température} $28^{\circ} \mathrm{C}$.

Les températures moyennes sont élevées; puisque la moyenne annuelle est supérieure à

Au cours de l'année, la température (minimums et maximums) s'élève progressivement de janvier à mai. Durant l'hivernage de juin à septembre, les vents pluvieux provoquent un abaissement des maximums et minimums. Après un nouveau maximum enregistré en octobre (fin des pluies), la température s'abaisse régulièrement jusqu'en décembre.

La nébulosité est faible en général, sauf durant la saison des pluies de juillet à septembre où elle est très élevée.

L'évaporation est intense, de l'ordre de $3,80 \mathrm{~m}$ à Linguère.

\section{La pluviométrie}

La station de Dahra se trouve approximativement sur l'isohyète $450 \mathrm{~mm}$.

La distribution et la quantité d'eau sont très variables d'une année à l'autre et ces différences se répercutent sur le disponible fourrager. Il faut noter d'autre part que, même dans un 
espace restreint comme la concession du C.R.Z., il y a d'une part des variations importantes d'intensité de la chute de pluie d'un point à un autre, et d'autre part la possibilité de chutes très localisées alors que le point voisin ne reçoit pas d'eau.

En ce qui concerne la distribution des pluies, il existe certaines années une période de sécheresse dite "intercalaire" (exemple 1972), c'est-à-dire qui intervient après une ou plusieurs chutes de pluies qui ont permis la germination des graines précoces. Cette sécheresse a pour conséquence la disparition presque complète des espèces précoces et par conséquent un changement très important de la qualité fourragère du pâturage.

\section{L'hygrométrie}

L'humidité relative est faible sauf durant les mois d'hivernage où elle peut dépasser 60 p. 100. Pour Dahra, la moyenne annuelle est d'environ 49 p. 100.

\section{Les vents}

Il existe deux orientations dominantes au cours de l'année :

- les alizés et l'harmattan, vents secs qui se rencontrent de novembre à mai;

- les vents correspondant à la mousson (Ouest) chargés de vapeur d'eau.

\section{LE MATERIEL ANIMAL: LE ZEBU GOBRA}

Le zébu Gobra est un animal bien musclé, subconvexiligne, longiligne, eumétrique. Les cornes sont en lyre haute, fortes à la base. Leur longueur peut atteindre 70 à $80 \mathrm{~cm}$. La robe est également blanche ou légèrement froment. Surtout chez les mâles, on trouve des bringeures et des charbonnures.

La bosse est développée surtout chez les mâles, en forme de bonnet phrygien dressé; chez la femelle, elle est plus petite et en forme de cône. L'animal est de grande taille : à 5-6 ans, $1,22 \mathrm{~m}$ chez les femelles et $1,33 \mathrm{~m}$ chez les mâles. La production laitière est faible mais suffit à peu près au veau et surtout est susceptible d'amélioration. Le rendement boucher est compris entre 50 et 53 p. 100.

Les animaux sont entretenus dans des conditions aussi proches que possible des conditions extérieures sans que les facteurs d'amélioration tels que l'absence de surpâturage, l'abreuvement à volonté, la surveillance sanitaire soient négligés.

En effet, chaque année, le Centre libère un certain nombre de mâles issus de son troupeau pour les introduire dans les troupeaux des éleveurs traditionnels. Il faut, par conséquent, que ces animaux conservent une bonne rusticité pour pouvoir résister valablement aux difficiles conditions extérieures, et donc à partir de 12 mois sont élevés uniquement en extensif sans supplémentation autre que minérale.

Par contre, de la naissance au sevrage qui a lieu entre 6 et 7 mois, les veaux reçoivent en plus du lait maternel, une supplémentation de $500 \mathrm{~g}$ par jour et par tête d'un concentré titrant 0,80 UF et $90 \mathrm{~g} \mathrm{M.A.D.} \mathrm{par} \mathrm{kg}$. A la fin de la saison sèche, les veaux sevrés reçoivent une supplémentation variable de 500 à $1.000 \mathrm{~g}$ du même concentré suivant l'avancement de la saison. Pour tous les animaux, l'abreuvement est à volonté.

Les femelles sont régulièrement mises à la reproduction vers $24-27$ mois; la monte est naturelle, libre, et jusqu'en 1969, il n'existait pas de saison de monte. Depuis, celle-ci a été placée du 15 août au 15 décembre de chaque année dans le but de regrouper les naissances à la période la plus favorable de l'année, et d'essayer de régulariser en diminuant leur durée les intervalles entre les vêlages. 
Les animaux ont été régulièrement pesés et mesurés suivant un protocole mis au point par l'I.E.M.V.T.: tous les veaux ont été observés toutes les semaines de la naissance à 3 mois, tous les 15 jours de 3 à 6 mois, tous les mois de 6 mois à 2 ans, tous les 3 mois de 2 à 6 ans et tous les 6 mois ensuite. Les observations effectuées sont le poids, le périmètre thoracique, la longueur scapulo-ischiale, la hauteur au garrot et aux sangles, la longueur et la largeur de la croupe, la longueur et la largeur de la tête.

\section{RESULTATS DES OBSERVATIONS ET MESURES}

\section{IV.1. Composants du niveau de fécondité du troupeau $(13,17)$}

\section{IV.1.1. Age au $1^{\text {er }}$ vêlage (4)}

L'âge au $1^{\text {er }}$ vêlage est un facteur important du niveau de fécondité. Chez les femelles Gobra, en brousse, le premier veau est obtenu entre 4 et 5 ans; 66 p. 100 des femelles ont vêlé à 4 ans et 89 p. 100 à 5 ans $(2,11)$. Au C.R.Z. de Dahra, l'âge moyen calculé sur 534 données est de $1365,6 \pm 24$ jours, soit environ 45 mois. Les femelles sont donc peu précoces, et on peut constater qu'il n'y a pas d'amélioration sensible au niveau de la station; ceci provient certainement du fait que les conditions d'entretien des animaux, bien que meilleures, sont encore volontairement proches de celles rencontrées à l'extérieur.

Un certain nombre de facteurs en relation avec cette donnée ont été étudiés. Ce sont les suivants :

1. Age au $1^{\mathrm{er}}$ vêlage en fonction du mois de naissance de la mère;

2. Influence du sexe du produit sur l'âge au premier vêlage;

3. Corrélation entre l'âge au premier vêlage et le poids du produit obtenu;

4. Influence de l'âge au $1^{\mathrm{er}}$ vêlage sur les intervalles entre les vêlages.

Les résultats sont les suivants:

1. L'influence saisonnière est importante d'une part sur la croissance, d'autre part sur l'évolution pondérale des animaux adultes. Il était par conséquent intéressant de savoir si cette influence de la saison sur le développement de la femelle pouvait avoir une conséquence sur la date de son entrée en reproduction. Les calculs effectués ont montré qu'en moyenne la période de vêlage n'intervient pas. Par contre, il existe une différence significative entre certains mois de l'année (janvier et avril). La cause de cette différence n'a pas été élucidée;

2. L'influence du sexe du produit n'existe pas sur le facteur considéré;

3. Il existe une corrélation positive entre l'âge au $1^{\text {trr }}$ vêlage et le poids de naissance du veau. En fait, ceci est dû au degré de maturité pondérale de la mère. Les veaux de génisses sont toujours plus légers et le poids des veaux augmente lentement au fil des gestations pour être maximal vers la $4^{\mathrm{e}}$ gestation;

4. Il n'y a pas de relation entre l'âge au $1^{\text {er }}$ vêlage et l'intervalle entre le $1^{\text {er }}$ et le $2^{\text {e }}$ vêlage. En effet, dans certaines races, précoces il est vrai, la femelle qui vêle trop tôt, met plus longtemps à récupérer ses possibilités physiologiques de fécondation; et par conséquent,

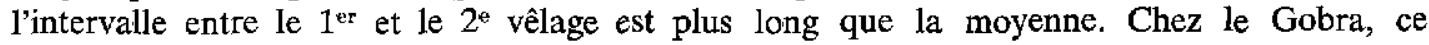
phénomène n'a pas été mis en évidence, car l'animal n'est pas précoce dans les conditions d'élevage traditionnel et même un vêlage un peu plus précoce dans les conditions naturelles n'a que peu d'action sur le déroulement ultérieur de la vie de reproduction. Par contre, entre l'âge au $1^{\text {er }}$ vêlage et la moyenne des intervalles entre les vêlages ultérieurs ( 2 à 8 ), il existe une relation significative, ce qui permet d'utiliser le critère âge au $1^{\text {er }}$ vêlage pour avoir une idée des qualités de reproductrice de la femelle. 


\section{IV.1.2. Intervalles entre les vêlages (5)}

Durant la vie d'une femelle, le nombre de veaux produits est d'une grande importance sur le plan de la productivité du troupeau. Bien que l'intervalle entre les vêlages soit une durée englobant plusieurs phénomènes successifs (durée de l'intervalle entre vêlage et première saillie fécondante, durée de la gestation), il constitue en lui-même un critère intéressant de la fertilité des femelles.

L'intervalle moyen calculé à partir de 1.254 observations est de 473 jours \pm 8 jours, soit environ 15 mois et demi. Il faut par conséquent attendre en moyenne 5 à 6 mois pour que la femelle Gobra qui a vêlé soit de nouveau fécondée. Ceci est le résultat obtenu en station. En brousse, cet intervalle est classiquement de 18 mois, mais lorsque les conditions climatiques sont mauvaises, ce qui est le cas depuis quelques années, l'intervalle devient plus long jusqu'à 22 mois $(2,11)$.

L'analyse de l'intervalle obtenu en station montre que dans seulement 39 p. 100 des cas, il est inférieur à 395 jours (soit 13 mois) et dans 44 p. 100 des cas, inférieur à 456 jours (15 mois), ce qui est une performance très faible. Les raisons évoquées pour expliquer la longue durée des intervalles sont :

1. La présence permanente des taureaux dans les troupeaux, ce qui entraîne, à cause de saillies trop précoces, une diminution de chances de fécondation des femelles;

2. Les causes alimentaires quantitatives, qualitatives;

3. La lactation.

Divers facteurs ont été étudiés dans leur relation avec les intervalles entre les vêlages. Ce sont les suivants :

1. Intervalles en fonction du mois de vêlage;

2. en fonction du sexe du produit;

3. en fonction des performances du produit précédent (effet de la lactation et de la gestation);

4. en fonction de l'année;

5. en fonction du numéro de vêlage;

6. liaison avec le poids du produit obtenu.

Les résultats de cette analyse sont les suivants:

1. Il n'existe pas de différence significative entre les différents mois (F00 $5=1,31<1,80$ ). Cependant, on peut noter que dans l'ensemble les valeurs des intervalles les plus importantes se rencontrent dans la période de fin d'hivernage et de post-hivernage;

2. Pas de différence significative;

3. Pas de corrélation pour l'effet de la gestation, c'est-à-dire pas d'influence du poids du veau de rang de vêlage $n$ sur la durée de l'intervalle à $n+1$;

Pas de corrélation pour l'effet de la lactation, c'est-à-dire de liaison entre le poids au sevrage du veau de rang $n$ sur la durée de l'intervalle $n$ à $n+1$;

4. Entre les différentes années, la différence est significative, mais il faut signaler que, les vêlages jusqu'en 1969 se produisant toute l'année, les femelles n'étaient pas placées dans des conditions similaires. L'installation de la saison de monte permettra de cerner mieux le problème;

5. Les différences entre les moyennes d'intervalles sont très significatives. Du $1^{\mathrm{er}}$ au $5^{\mathrm{e}}$ intervalle, il y a une diminution très nette de la valeur des intervalles (19 p. 100). Ensuite, il y a une légère remontée, puis la valeur se stabilise;

6. Il existe une corrélation positive et significative entre l'intervalle du vêlage $n$ au vêlage $n+1$ et le poids du produit obtenu. Plus lintervalle est long et plus la femelle a la possibilité de reconstituer ses réserves et de mener à bien une nouvelle gestation. 
IV.1.3. Installation de la saison de monte (6)

L'analyse d'un certain nombre de facteurs dont:

- Ia répartition naturelle des vêlages;

- l'évolution pondérale des mères;

- l'évolution pondérale des produits;

- l'intervalle entre les vêlages;

- la mortalité des produits en fonction de la période de naissance;

- l'organisation et le mode d'entretien du troupeau...

a permis de mettre en place une saison de monte située du 15 août au 15 décembre de chaque année.

L'intérêt de la saison de monte est très important car elle permet de regrouper pendant une seule période l'ensemble des naissances. Ainsi les animaux seront-ils tous placés dans des conditions identiques, ce qui est important par exemple dans le domaine du testage et de la comparaison des taureaux. D'autre part, les opérations telles que les vaccinations, les supplémentations, les sevrages peuvent être appliquées toujours à la même période et en un nombre minimal d'opération.

Cette saison de monte est appliquée depuis 1969 à Dahra et actuellement 91 p. 100 des naissances (sur 100 naissances en 1972) ont lieu pendant la saison de monte. En effet, par exemple, en 1972, 9 naissances ont eu lieu en dehors de la saison programmée en raison du faible nombre de parcs à la disposition des animaux, donc de leur proximité, donc du passage par les mâles des clôtures pour rejoindre les femelles.

Malheureusement, la climatologie extrêmement mauvaise des dernières années n'a pu permettre de mettre en évidence les bienfaits de l'opération.

\section{IV.2. Autres problèmes étudiés}

\section{IV.2.1. Durée de la gestation $(14,15)$}

La durée de la gestation est étudiée depuis trois années au C.R.Z. de Dahra où les saillies d'une part et d'autre part les vêlages sont relevés systématiquement.

La durée actuellement déterminée sur 183 observations est de 293 jours \pm 2 jours.

La durée n'est pas significativement différente selon que les produits obtenus sont des mâles ou des femelles $(F=0,47)$.

Les données selon le sexe se présentent comme suit:

\section{TABLEAU $\mathrm{N}^{\circ} \mathrm{I}$}

\begin{tabular}{|l|c|c|}
\hline & Males & Femelles \\
\hline Nombre d'observations & 787 & 96 \\
Moyenne & 293,71 & 292,63 \\
Intervalle de confiance & $\pm 2,14$ & $\pm 2,23$ \\
\hline
\end{tabular}

Erratum: 1re ligne, au lieu de 787, lire 87. 


\section{IV.2.2. Age de la puberté chez les femelles $(14,15)$}

A l'occasion de l'étude du rythme des chaleurs et de la période interoestrale, les jeunes femelles sont placées dès 12 mois avec un mâle vasectomisé chargé de détecter les chaleurs éventuelles. Les résultats sont encore très peu nombreux (26) mais suffisent à reconnaître le manque de précocité de la femelle Gobra dans les conditions naturelles. En effet, l'âge moyen auquel sont suivies les premières chaleurs est de 26 mois, ce qui correspond aux observations sur l'âge au $1^{\text {er }}$ vêlage.

Ce manque de précocité peut être éliminé si la femelle bénéficie d'une alimentation correspondant à ses besoins. L'expérience d'extériorisation des potentialités génétiques des femelles a permis de montrer que l'âge au $1^{\text {er }}$ vêlage passait de $1.077 \pm 99$ jours à $900 \pm 26$ jours, soit une différence de 6 mois environ. Ce point sera repris dans un prochain chapitre (IV.4.).

\section{IV.2.3. Etude des chaleurs (intervalle - durée) $(14,15)$}

Depuis le début de l'année 1971, toutes les génisses nées au C.R.Z. sont placées à partir de 12 mois dans le troupeau d'étude de sexualité. Les chaleurs sont détectées à l'aide de deux taureaux à pénis dévié selon la méthode de ROMMEL (1960). La durée moyenne calculée sur 150 observations retenues à ce jour est $21,5 \pm 0,5$ jours.

Un problème intéressant concerne la question de la pérennité du cycle tout au long de l'année. Il s'agit de savoir si les chaleurs présentent une interruption saisonnière. Les données en notre possession sont encore en nombre trop faible pour procéder à une analyse systématique; on peut cependant, en examinant la fréquence des chaleurs observées en fonction du mois, avoir une idée de la physionomie du phénomène.

Sur 150 observations effectuées, on s'aperçoit qu'au cours de l'année il n'y a pas d'interruption de la vie sexuelle des femelles mais on note cependant une diminution de l'activité durant la fin de la saison sèche. La période d'activité la plus intense est observée aux mois d'août et septembre, ce qui confirme les données obtenues par ailleurs, à savoir l'époque maximale des naissances (juin et juillet (6) aussi bien au C.R.Z. qu'à l'extérieur (2-11). Une seconde période d'activité est observée mais incomparablement moins intense en fin mars - début avril. Elle est due probablement à des modifications de l'alimentation animale (cf. montée de la sève et renouveau de la végétation arborée à cette époque).

En ce qui concerne la durée, on distingue la période pro-œstrale, durant laquelle le taureau suit la femelle et tente de la saillir sans que celle-ci accepte: cette période dure entre 9 et 10 heures pour 75 observations. Les manifestations d'inquiétude, d'essais de saut sur les autres femelles apparaissent plus tôt.

La duré de la période cestrale pendant laquelle la femelle accepte la saillie dure en moyenne de 5 à 6 heures (75 observations).

Enfin, le nombre de saillies acceptées par la femelle au cours de l'œstrus est très variable. La moyenne est de 4,8 saillies mais les extrêmes relevés vont de 1 à 23 sauts acceptés.

Les chaleurs sont très peu visibles chez les animaux courants; par contre chez les animaux supplémentés soit en totalité (lot d'extériorisation), soit en matières minérales seulement (30 $\mathrm{g} / \mathrm{j} /$ tête de phosphate bicalcique puis à volonté sous forme de pierres à lécher) les manifestations classiques de l'état de rut sont beaucoup plus fréquemment signalées.

\section{IV.2.4. Etude de l'involution utérine $(14,15)$}

Parmi les composants du niveau de fécondité appréciés par l'intervalle moyen entre les vêlages, la durée de l'involution utérine constitue une constante qu'il convient de connaître. 
Pour ce faire, en 1972, les utérus de toutes les vaches Gobra ont été systématiquement et régulièrement palpés deux fois par semaine après le part.

La durée de cette involution calculée sur 83 observations est de $29 \pm 1$ jours, chiffre qui correspond assez parfaitement aux données recueillies dans les autres races.

La période d'involution utérine constitue une partie de la "période de service » qui est l'intervalle compris entre la parturition et la nouvelle fécondation. Elle est constante et par conséquent la dernière période à analyser est celle comprise entre le retour à la normale de l'utérus et la nouvelle fécondation, la durée de la gestation étant connue.

Chez le Gobra, cette durée est donc environ de 150 jours soit 5 mois. C'est durant cette dernière période qu'interviendront tous les obstacles à la fécondation, hormonaux (en particulier lactation) et nutritionnels.

\section{IV.2.5. Essais de synchronisation de l'astrus}

La synchronisation de l'œstrus doit permettre :

- la prévision de l'cestrus;

- de rendre celui-ci plus apparent;

- de rendre possible l'insémination simultanée d'un groupe important d'animaux.

Trois essais ont jusqu'à présent été pratiqués au C.R.Z. de Dahra.

Les deux premiers ont fait appel à l'utilisation de la Norethandrolone pendant 10 jours avec des injections de valérate d'œstradiol le premier jour, et de PMSG le dernier jour.

Malheureusement l'opération s'est soldée par un échec sur le plan de la fécondation. Les raisons en sont probablement :

- la jeunesse et le manque de précocité des femelles;

- l'alimentation précaire dont elles ont pu bénéficier;

- l'inexpérience des opérateurs chez ces animaux;

- les effets propres dus aux ostrus induits.

Les chaleurs ont été systématiquement contrôlées dans leur apparition par l'utilisation d'un taureau à pénis dévié selon la technique de ROMMEL. 70 p. 100 des femelles ont été synchronisées: $48 \pm 8$ heures après l'arrêt du traitement.

Lorsque l'insémination artificielle est pratiquée à la $60^{\mathrm{e}}$ heure, 65 p. 100 des femelles sont en chaleur depuis 12 heures, ce qui normalement constitue le bon moment pour l'insémination.

Le $3^{e}$ essai a été pratiqué à l'aide de méthyl-6 $\alpha$ acetoxy - $17 \alpha$ Progesterone (Specia Fartutal) qui s'administre par voie orale à raison de $180 \mathrm{mg}$ par jour pendant 18 jours. Les résultats sont beaucoup moins intéressants puisque seulement 44,5 p. 100 des femelles ont été synchronisées de manière détectable par le taureau, en moyenne 43 heures (39-50) après l'arrêt du traitement blocant. Le traitement par voie orale donne donc des résultats moins valables que ceux obtenus par voie parentale mais présente l'immense avantage d'une plus grande facilité d'application pratique. Les travaux dans ce domaine vont être activement poursuivis.

La synchronisation chimique paraît donc d'application relativement difficile du moins avec les produits expérimentés jusqu'alors.

Mais il faut signaler et nous l'avons vu dans les chapitres précédents, que le milieu naturel se charge d'une synchronisation qui, pour n'être pas regroupée dans un laps de temps très court, n'en est pas moins effective.

En effet, l'explosion des capacités de fécondation des animaux après l'hivernage traduit bien 
l'action très importante du flushing alimentaire qui s'applique à l'ensemble des vaches dont une forte proportion est fécondée durant une période courte.

\section{IV.3. Influence de quelques facteurs sur les paramètres de fécondité}

\section{IV.3.1. Influence de la saison et de la nutrition $(7,8)$}

Ces deux facteurs ont été regroupés parce qu'en fait sont indissolublement liés. En effet, les diverses variations saisonnières observées s'expriment essentiellement par une plus ou moins grande disponibilité alimentaire aussi bien sur le plan qualitatif que sur le plan quantitatif et ont des conséquences physiologiques très importantes. Sur le plan de l'évolution pondérale des animaux adultes, il a été montré que des variations saisonnières importantes existent. Ces variations peuvent atteindre jusqu'à 20 p. 100 du poids des animaux si l'on compare le poids de ceux-ci au cours des mois les plus favorables (novembre, décembre) et des mois les plus défavorables (juin et surtout juillet). Il a été montré aussi que la vie de reproduction a une influence importante sur cette évolution pondérale (9). En effet, les pertes de poids les plus sensibles sont enregistrées pour les femelles vêlant au cours du premier et du deuxième trimestres. A la fin de l'année, seules les vaches vêlant aux troisième et quatrième trimestres ont un poids supérieur à celui qu'elles avaient au début de l'année.

De même au niveau de la croissance, l'effet saisonnier se fait fortement sentir, elle est véritablement en dents de scie et, suivant le mois de naissance, les résultats pondéraux aux différents âges sont très variés (8).

Cette évolution saisonnière de la vie des animaux se retrouve au niveau de la répartition des naissances; celles-ci, comptabilisées depuis 1955, ont été réparties par mois. Les résultats apparaissent sur le tableau $\mathbf{n}^{\circ}$ II. Aux mois de mai, juin et juillet, ont lieu environ 46 p. 100 des naissances. En 1968, les saillies ont été suivies pendant toute l'année et les résultats présentés au tableau $n^{\circ}$ III corroborent ceux du tableau précédent, à savoir que les mois d'août, de septembre à novembre sont les plus propres à la conception (6).

\begin{tabular}{|c|c|c|}
\hline \multicolumn{3}{|c|}{ TABLEAU $N^{\bullet}$ II } \\
\hline Mois & Nombre & p. 100 \\
\hline 1 & 153 & 5,56 \\
2 & 228 & 8,29 \\
3 & 232 & 8,43 \\
4 & 183 & 6,65 \\
5 & 321 & 11,67 \\
6 & 473 & 17,20 \\
7 & 464 & 16,87 \\
8 & 243 & 8,83 \\
9 & 159 & 5,78 \\
10 & 91 & 3,30 \\
11 & 92 & 3,34 \\
12 & 111 & 4,03 \\
\hline Tota1 & 2.750 & 100 \\
\hline
\end{tabular}

\begin{tabular}{|c|c|c|}
\hline \multicolumn{3}{|c|}{ TABLEAU N $^{\bullet}$ III } \\
\hline Mois & Nombre & p.100 \\
\hline 1 & 6 & 4,87 \\
2 & 0 & - \\
3 & 1 & 0,81 \\
4 & 15 & 12,19 \\
5 & 9 & 7,31 \\
6 & 9 & 7,31 \\
7 & 5 & 4,06 \\
8 & 6 & 4,87 \\
9 & 23 & 18,69 \\
10 & 26 & 21,13 \\
11 & 20 & 16,26 \\
12 & 3 & 2,4 \\
\hline Tota1 & 123 & 100 \\
\hline
\end{tabular}

Le caractère saisonnier des saillies fécondantes est donc très net au niveau du troupeau. Sans qu'il y ait semble-t-il un anoestrus saisonnier véritable, on assiste cependant à une véritable explosion des possibilités de fécondation au moment où les femelles ont retrouvé un équilibre physiologique correct après le début de l'hivernage.

Les différents composants de la fécondité précédemment traités dans ce rapport ne seront pas repris en détail dans l'optique de l'étude de l'influence saisonnière, mais on peut rappeler que la saison et ses conséquences nutritionnelles agissent au niveau de l'intervalle entre les vêlages au cours de la même année et d'une année à l'autre, sur le taux de fécondité et de sevrage (67 à 81 p. 100) (7). 
Pour vérifier l'action du facteur alimentaire sur les performances pondérales des femelles Gobra, une expérience a été menée visant à éliminer ce facteur limitant en distribuant à un lot de 28 femelles un concentré équilibré distribué à volonté depuis leur naissance $(14,15)$.

Les femelles ont été placées à la reproduction accompagnées de lots témoins non supplémentés en janvier et août 1970, soit à un âge moyen de 18 et 24 mois respectivement.

Les premiers résultats concernent l'âge au premier vêlage. Ils sont les suivants :

- Lots supplémentés: 96 p. 100 des femelles ont vêlé (1 femelle stérile maintenant éliminée à 4 ans et 3 mois) - à 925 et 940 jours.

— Lots témoins: 100 p. 100 des femelles ont vêlé à 1.100 et 1.260 jours.

Le gain est donc conséquent. Il faut noter qu'en moyenne, on a intérêt à placer les femelles assez tôt à la reproduction, même chez les animaux non supplémentés. Cette opération permettant de gagner une année sur la production de certains animaux.

En ce qui concerne l'intervalle entre les vêlages, les résultats s'énoncent comme suit :

- Lot supplémentés : 100 p. 100 des femelles ayant vêlé une première fois ont eu un second veau, et 45,4 p. 100 un troisième veau.

Intervalle $1-2=384$ jours.

Intervalle $2-3=351$ jours.

- Lots témoins : 83 p. 100 des femelles ayant eu un veau en ont eu un second et 0 p. 100 un troisième.

Intervalle $1-2=403$.

A la lumière de ces données, il apparaît comme certain que la femelle Gobra, lorsque son environnement devient propice, possède une précocité très correcte et des intervalles entre les vêlages inférieurs à 395 jours, ce qui constitue une excellente performance. Cette expérience permet aussi de montrer que lorsque les conditions alimentaires mises à la disposition des animaux sont normales, l'influence saisonnière disparaît et ces femelles sont fécondables, et fécondées, quelle que soit la période de l'année envisagée.

\section{IV.3.2. Influence de la production laitière}

La production laitière intervient au niveau des intervalles entre les vêlages. Les analyses effectuées à ce sujet montrent que si la lactation n'intervient pas sur le plan quantitatif du fait des capacités laitières très faibles des femelles de la race, elle intervient certainement sur le plan qualitatif, par sa seule présence, qui agit sur le rétablissement de la fonction ovarienne. En effet, tant que le veau est sous la mère (sevrage entre 6 et 7 mois) en moyenne la fécondation ne se produit pas. Il est probable d'ailleurs que, là aussi, l'influence nutritionnelle soit le facteur causal essentiel du phénomène (7).

\section{QUELQUES INFORMATIONS SUR LES MALES}

Actuellement au C.R.Z. de Dahra, un certain nombre de jeunes mâles sont cédés à des éleveurs locaux afin d'améliorer la production moyenne de leurs troupeaux. Malheureusement, la demande est assez forte et par conséquent la qualité moyenne des reproducteurs cédés, d'ailleurs non testée sur la descendance, est d'autant plus faible que le nombre d'animaux cédés est plus grand. C'est pourquoi l'implantation de l'insémination artificielle doit être une préoccupation importante. Il convient donc de dresser des mâles pour la monte. Les opérations sont en cours et ne présentent pas de difficultés essentielles, mis à part le fait que ces mâles ne semblent pas posséder une ardeur particulièrement remarquable. Actuellement, une dizaine de taureaux est dressée. 
Les projets en cours consistent à effectuer un prétestage des jeunes animaux qui comprend une étude de leur capacité de croissance à moindre frais, une étude de leur spermatogénèse et un dressage concomitant.

Les résultats actuels concernant le sperme récolté sont les suivants :

\begin{tabular}{|l|c|}
\hline \multicolumn{2}{|c|}{ TABLEAU $N^{\circ} \mathrm{IV}$} \\
\hline $\mathrm{pH}$ & 6,1 \\
\hline Morta1ité & 4 \\
\hline Volume (m1) & 2,40 \\
\hline Concentration & 1300 000 \\
\hline Anomalies (p.100) & 8 \\
\hline Morts (p.100) & 10 \\
\hline Réductase (mn) & 1,3 \\
\hline
\end{tabular}

Erratum : 2e ligne, au lieu de Mortalité, lire Motilité.

\section{CONCLUSIONS}

Les données concernant la reproduction sur le zébu Gobra permettent actuellement de se faire une idée assez précise de la vie sexuelle des femelles.

Il apparaît très nettement que le comportement de ces animaux est très fortement tributaire des facteurs bioclimatiques dont l'action se traduit essentiellement au niveau des disponibilités alimentaires qui à leur tour conditionnent les réponses physiologiques des animaux. La reproduction fait partie des activités productives qui sont réduites lorsque les possibilités alimentaires qualitatives et quantitatives se trouvent réduites.

Par contre, si l'animal se trouve placé dans des conditions normales d'existence, il extériorise des possibilités tout à fait remarquables sur les plans de la production de veaux et de viande.

C'est-à-dire que les opérations de sélection effectuées sur ces animaux peuvent se poursuivre sans appréhension, mais aussi qu'elles doivent obligatoirement s'accompagner d'une amélioration du cadre de vie (alimentation et abreuvement en particulier).

\section{SUMMARY}

Survey of breeding characteristics in Gobra zebu cattle at Dahra zootechnical research Center (Senegal)

In the report, some characteristics of the reproduction of Gobra zebu cattle are related. The authors have studied age at first calving, intervals between calvings, problems to be solved for a rational breeding season, lenght of gestation, age at puberty, lenght of heats and intervals between them, uterine involution and synchronization of estrus.

Studies on the influence of nutrition, lactation and epoch of the year in the process of reproduction are also considered. At last, some data on reproductive qualities of males are given.

\section{RESUMEN}

Características de la reproducción en el cebú estudiadas en el Centro de investigaciones zootécnicas de Dahra (Senegal)

Los autores hacen el balance sobre las varias características actualmente conocidas de la reproducción en el cebú Gobra. 
Se analizan sucesivamente la edad al primer parto, el intérvalo entre los partos, los problemas planteados por la instalación de una estación de apareamiento, la duración de la gestación, la edad de la pubertad, el celo (intervalos y duración), la involución uterina, el problema del dominio del ciclo estral. Luego se estudian ciertos factores influyendo en la vida de reproducción, es decir la estación, la nutrición y la lactación.

Se dan tambien algunos informes a propósito de los machos.

\section{BIBLIOGRAPHIE}

1. BERTRAND (M.). Les causes générales de la subfertilité animale. Numéro spécial de reproduction. Bull. Techn. Inf., 1971 (257): 109-114.

2. COSTIOU (P.). Rapport d'enquête sur le cheptel bovin du Ferlo. Dakar, I.E.M.V.T. Région de Recherches vétérinaires et zootechniques de l'Ouest africain, 1972, Rapport ronéo. 40 p., 17 tabl.

3. COUROT (M.), GOFFAUX (M.), ORTAVANT (R.). Analyse des variations saisonnières de la fertilité des bovins dans le Jura français. Ann. Biol. anim. Bioch. Biophys., 1968, 8 (2) : 209-216.

4. DENIS (J.P.). Note sur l'âge du 1er vêlage chez le zébu Gobra. Communication à la Conférence internationale de Zootechnie. Versailles, juillet 1971.

5. DENIS (J. P.). L'intervalle entre les vêlages chez le zébu Gobra. Rev. Elev. Méd. vét. Pays trop., 1971, 24 (4): 635-647.

6. DENIS (J.P.). Note sur les facteurs conduisant au choix d'une saison de monte au C.R.Z. de Dahra (à paraître).

7. DENIS (J. P.). Influence des facteurs bioclimatiques sur la reproduction des femelles Zébu en milieu tropical sec. Communication au VIIe Congrès de reproduction animale et d'insémination artificielle. Munich, 6 au 9 juin 1972.

8. DENIS (J. P.), VALENZA (J.). Etude et sélection du zébu Peulh sénégalais (Gobra). Communication au Congrès mondial de production animale. Université de Maryland (U.S.A.), juillet 1968.

9. DENIS (J. P.), VALENZA (J.). Comportement pondéral des vaches adultes de race Gobra. Comparaison avec les animaux importés Pakistanais et Guzera. Rev. Elev. Méd. vét. Pays trop., 1970, 23 (4): $229-241$.

10. DENIS (J.P.), VALENZA (J.). Etude de la mortalité bovine au C.R.Z. de Dahra. Rev. Elev. Méd. vét. Pays trop., 1972, 25 (4) : 445-453.

11. FAYOLLE (F.). Rapport d'enquête sur le cheptel bovin. Région d'Amaly. Dakar, I.E.M.V.T., août 1972. Rapport ronéo. 24 p., 3 graph., 13 tabl.

12. NAEGELE (A. G.). Etude des pâturages naturels de la forêt classée des six forages ou réserve sylvopastorale du Koya (République du Sénégal). Tome I: Généralités sur la région étudiée. Rome, F.A.O., 1971. (Etude no 4: Pâturages et cultures fourragères).

13. ORTAVANT (R.), COUROT (M.) et FREBLING (J.). Etude des paramètres de fécondité des troupeaux bovins. Numéro spécial reproduction. Bull. Techn. Inf., 1971 (257): 81-87.

14. Rapports annuels de la Région de Recherches vétérinaires et zootechniques de l'Ouest Africain. I.E.M.V.T., Zootechnie, 1954 à 1972.

15. Rapports annuels du C.R.Z. de Dahra-Djoloff. Section de Recherches. 1954 à 1972.

16. RAYNAL (J.). Etude botanique des pẫturages du C.R.Z. de Dahra-Djoloff, Sénégal. Paris, O.R.S.T.O.M., 1964.

17. VISSAC (B.) et POLY (J.). Etude statistique des causes de variation de quelques paramètres du cycle de reproduction des vaches laitières. L'intervalle vêlage-fécondité dans les troupeaux de Seine-et-Marne. Ann Zootechn., 1957, $6:$ :237-268. 